\title{
Editorial - Preprint e postprint em publicações científicas e no Direito: discussões e medidas para ciência aberta e divulgação de pesquisas
}

\author{
Editorial - Preprint and postprint in scientific publications \\ and in Law: discussions and measures to open science \\ and research communication
}

\section{Vinicius Gomes de Vasconcellos ${ }^{\mathbf{1}}$}

Universidade Estadual de Goiás, Goiânia, Goiás, Brasil Instituto Brasileiro de Ensino, Desenvolvimento e Pesquisa IDP, Brasília, Distrito Federal, Brasil vinicius.vasconcellos@ueg.br lattes.cnpq.br/9628659956663949 orcid.org/0000-0003-2020-5516

p publons.com/a/1174099/

\section{Felipe da Costa De-Lorenzi ${ }^{2}$}

Pontifícia Universidade Católica do Rio Grande do Sul, Porto Alegre, Rio Grande do Sul, Brasil felipe.lorenzi91@gmail.com http://lattes.cnpq.br/8132922711654157 https://orcid.org/0000-0003-4979-5644

p https://publons.com/a/3399489/

1 Doutor em Direito pela Universidade de São Paulo, com período de sanduíche na Universidad Complutense de Madrid/ESP (bolsa PDSE/CAPES) e estágio de pós-doutoramento pela Universidade Federal do Rio de Janeiro. Mestre em Ciências Criminais pela PUCRS. Professor permanente do Instituto Brasileiro de Ensino, Desenvolvimento e Pesquisa - IDP/DF (mestrado/doutorado). Professor efetivo da Universidade Estadual de Goiás. Editor-chefe da RBDPP. Assessor de Ministro no Supremo Tribunal Federal.

2 Doutor e Mestre em Ciências Criminais pela Pontifícia Universidade Católica do Rio Grande do Sul, com período sanduíche na Humboldt-Universität zu Berlin (bolsas PDSE/CAPES e CDEA/DAAD). Editor-assistente da RBDPP. Advogado. 
Resumo: Recentemente, a Revista Brasileira de Direito Processual Penal alterou suas políticas editoriais para, em conformidade com as diretrizes de Scielo e COPE, autorizar e incentivar o depósito de manuscritos em servidores certificados de preprint e postprint, de modo a ampliar a publicidade, a transparência e a celeridade na divulgação dos resultados de pesquisas científicas. Neste editorial, a partir de revisão bibliográfica, busca-se examinar alguns aspectos dessas práticas científicas, como sua origem, definição, benefícios e problemas, com foco na utilização nas ciências jurídicas - em particular, as criminais. Conclui-se que a tendência de ampliação do uso de preprints coloca novas perspectivas à produção de conhecimento e à editoração de revistas científicas, contribuindo com a consolidação de medidas para uma ciência aberta. Seus riscos e benefícios devem ser ponderados com cautela, mas as potenciais vantagens se sobressaem, como, por exemplo, a maior celeridade na divulgação científica e a possibilidade de maior debate para aprimoramentos na pesquisa até sua publicação definitiva.

PalaVRAs-ChaVe: editorial; preprint; postprint; divulgação científica; ciência aberta.

ABSTRACT: Recently, the Brazilian Journal of Criminal Procedure has changed its editorial policies to allow and encourage the submission of manuscripts in preprint and postprint servers, in accordance with guidelines from COPE and Scielo. The goal of this modification is to improve the publicity, transparency and celerity of scientific results communication. Based on bibliographical review, the current editorial aims to analyze some aspects of this scientific practice, such as its origin, definition, benefits and problems, focusing on the Legal Sciences - especially the Criminal Sciences. The conclusion is that the increased use of preprints provides new perspectives to the scientific production and editing, contributing to the consolidation of open science practices. The risks and benefits must be carefully weighted, but the potential advantages of preprints - such as faster dissemination of results and the possibility of wider debate and research improvement before the definitive publication - prevail.

KEY-WORDS: editorial; preprint; postprint; science communication; open science. 


\section{INTRODUÇÃO}

Sem dúvidas, a pandemia de Covid-19 acarretou impactos na ciência e na vida em geral. Durante este período, todas as atenções voltaram-se às descobertas científicas sobre tratamentos, vacinas etc., ressaltando a importância da pesquisa e da produção de conhecimento sério e consistente para a vida humana. Certamente, muitos artigos publicados aportaram contribuições para o aprimoramento da resposta à doença, $\mathrm{o}$ que potencialmente salvou um número incalculável de vidas.

Assim, considerando a importância de respostas rápidas a problemas urgentes, muitas pesquisas foram inclusive divulgadas antes de serem submetidas à análise formal em revistas científicas com controle por pares. Pessoas que acompanharam notícias, podcasts e outros meios perceberam uma expansão da divulgação científica e certamente devem ter notado avisos como "esta pesquisa ainda não foi submetida ao controle por pares” ou "esta é uma versão preprint, ainda não avaliada por outros pesquisadores".

Também no Direito há questões urgentes, sobre as quais a produção científica poderia contribuir para a solução ou resposta social mais adequada. Podemos citar, por exemplo, um julgamento de caso importante nos Tribunais, a ocorrência de mudanças normativas e eventuais dúvidas sobre a sua aplicação prática pelos juízos etc. Certamente, a divulgação mais rápida de pesquisas científicas poderia aportar importantes dados e conhecimentos para a resolução dos problemas jurídicos e conflitos sociais regulados pelo Direito.

Diante desse cenário, a Revista Brasileira de Direito Processual Penal alterou recentemente suas políticas editoriais para, em conformidade com as diretrizes de Scielo e COPE, ${ }^{3}$ autorizar e incentivar o depósito de manuscritos em servidores certificados de preprint e postprint, de modo

3 SCIELO Brasil, Critérios, política e procedimentos para a admissão e a permanência de periódicos na Coleção SciELO Brasil, versão maio 2020, p. 29. Disponível em: <https://wp.scielo.org/wp-content/uploads/20200500-Criterios-SciELO-Brasil.pdf>. Acesso em: 16 set. 2020; COPE Council. COPE Discussion document: Preprints. Mar. 2018, p. 2. Disponível em: <https:// publicationethics.org/resources/discussion-documents/preprints $>$. Acesso em: 16 set. 2020 . 
a ampliar a publicidade, a transparência e a celeridade na divulgação dos resultados de pesquisas científicas. ${ }^{4}$ Neste editorial, buscamos examinar alguns aspectos dessas práticas e premissas, como sua origem, definição, benefícios e problemas, com foco na utilização nas ciências jurídicas - em particular, as criminais.

O preprint não é uma ideia nova. A publicização de versões prévias de artigos tornou-se comum entre físicos nos anos 80, especialmente os pesquisadores de física teórica e física de altas energias, que compartilhavam o conteúdo dos manuscritos por correio, depois e-mail e, por fim, no site arXiv, fundado em 1991. ${ }^{5} \mathrm{Na}$ subárea de física de altas energias, um estudo de 2009 concluiu que, desde 1999, mais de $90 \%$ dos artigos publicados nos principais periódicos também foram disponibilizados, antes ou após a revisão por pares, no arXiv. ${ }^{6}{ }^{7}$ Também nas áreas de matemática e economia, há tempos existe uma cultura de preprints. ${ }^{8}$

Mesmo antes disso, ainda em 1961, o Instituto Nacional da Saúde dos Estados Unidos (National Institutes of Health) tentara criar uma plataforma de preprint - chamada Information Exchange Groups -, a qual acabou fechada em 1967 em razão de periódicos se recusarem a publicar

4 Disponível em: <http://www.ibraspp.com.br/revista/index.php/RBDPP/ about/editorialPolicies\#custom-8>. Acesso em: 18 set. 2020.

5 STERLING, Evan. The Rising Popularity of Preprints. In: WATSON, Jada (ed.). Moving through the Grey: Publishing in Action. Ottawa, 2018, p. 49. Disponível em: <https://ojs.scholarsportal.info/ottawa-school/index.php/ PA/issue/view/18>. Acesso em: 02/09/2020; LUTHER, Judy. The Stars Are Aligning for Preprints. The Scholarly Kitchen, 18 de abril de 2017. Disponível em: <https://scholarlykitchen.sspnet.org/2017/04/18/stars-aligning-preprints/>. Acesso em: 18 set. 2020.

6 GENTIL-BECCOT, Anne; MELE; Salvatore; Brooks, Travis C. Citing and Reading Behaviours in High-Energy Physics. Scientometrics, v. 84, n. 2, p. 345355, ago. 2010, p. 347-8.

7 Atualmente o arXiv atende as áreas de física, matemática, ciências da computação, biologia quantitativa, economia e outras disciplinas afins, sendo considerado o maior servidor do gênero no mundo. Cf. HOY, Matthew B. Rise of the Rxivs: How Preprint Servers are Changing the Publishing Process. Medical Reference Services Quarterly, v. 39, n. 1, p. 84-89, fev. 2020, p. 85.

8 STERLING, Evan. The Rising Popularity of Preprints. In: WATSON, Jada (ed.). Moving through the Grey: Publishing in Action. Ottawa, 2018, p. 49. Disponível em: <https://ojs.scholarsportal.info/ottawa-school/index.php/PA/ issue/view/18>. Acesso em: 02/09/2020. 
os artigos que circularam anteriormente na comunidade. ${ }^{9}$ No entanto, aproximadamente desde 2013 verifica-se uma "explosão de plataformas e serviços de preprint". ${ }^{10}$ Um estudo listou 18 servidores surgidos entre 2013 e 2018, criados por comunidades de diversas áreas, países, editoras e instituições de financiamento de pesquisas. ${ }^{11}$ Um dos primeiros nessa nova onda foi o bioRxiv, criado em 2013 pelo Cold Spring Harbor Laboratory para as ciências da vida. ${ }^{12}$ Também a famosa editora Nature criou um servidor de preprint em 2007 - chamado Nature Precedings - o qual, contudo, foi encerrado em $2012 .{ }^{13}$

Atualmente, existem servidores de preprint para quase todas as áreas ${ }^{14}$, inclusive o direito, como Scielo Preprints (opção preferencial recomendada pela RBDPP), LawArXiv, SocArXiv e SSRN. ${ }^{15}$ Muitos deles podem ser pesquisados no agregador criado pela Open Science Foundation, chamado OSF Preprints. É fundamental verificar a confiabilidade do

9 COBB Matthew. The prehistory of biology preprints: a forgotten experiment from the 1960s. PLoS Biology, v. 15, n. 11, nov. 2017, n.p.

10 TENNANT, Jonathan; BAUIN, Serge; JAMES, Sarah; KANT, Juliane. The evolving preprint landscape: Introductory report for the Knowledge Exchange working group on preprints. MetaArXiv (preprint), maio 2018.

11 TENNANT, Jonathan P. et al. Ten Hot Topics around Scholarly Publishing. MDPI, v. 7, n. 2, jun. 2019.

12 CHIARELLI Andrea; JOHNSON, Rob; PINFIELD, Stephen; RICHENS, Emma. Preprints and Scholarly Communication: An Exploratory Qualitative Study of Adoption, Practices, Drivers and Barriers [version 2]. F1000Research, p. 1-74, nov. 2019, p. 3.

13 Sobre isso, ver: <https://www.nature.com/npre/about>. Acesso em: 18 set. 2020.

14 HOY, Matthew B. Rise of the Rxivs: How Preprint Servers are Changing the Publishing Process. Medical Reference Services Quarterly, v. 39, n. 1, p. 84-89, fev. 2020, p. 85.

15 Vale citar também o F1000 Research, que apresenta, em realidade, uma proposta mais ampla, caracterizando um meio de publicação inovador, em que o artigo é disponibilizado publicamente desde a submissão, passando por controle por pares aberto com a publicação dos pareceres junto ao artigo, rodadas de correções públicas e a divulgação das novas versões corrigidas do manuscrito até a sua aprovação final, quando ocorre o depósito em indexadores como PubMed, Scopus e Google Scholar. Sobre isso, ver: <https:// f1000research.com/about>. Acesso em: 18 set. 2020. 
servidor, visto que diversos requisitos devem ser cumpridos e controlados. ${ }^{16} \mathrm{O}$ manuscrito não será considerado um preprint se disponibilizado em sites não certificados para tanto.

Apesar dessa clara tendência de adoção generalizada de preprints, é preciso discutir suas vantagens e desvantagens a fim de verificar se a prática deve ou não ser admitida e incentivada. Esse exame deve levar em consideração as particularidades de cada disciplina, pois o balanço entre prós e contras pode ser alterado a depender das específicas metodologias e lógicas de produção e divulgação do conhecimento. ${ }^{17}$

\section{O QUE SÃO PREPRINT E POSTPRINT? DISCUSSÕES SOBRE OS MOTIVOS PARA A SUA IMPLEMENTAÇÃO E POSSÍVEIS PONTOS POSITIVOS}

A definição de preprint é objeto de debates, de modo que inexiste conceito completamente aceito. ${ }^{18}$ As tentativas de definição de preprint envolvem relações com diversos elementos e discussões pertinentes. Chiarelli et al. identificaram seis fatores utilizados por diferentes autores a fim de defini-los: ${ }^{19}$

a) Gênero do trabalho. Normalmente se trata de um artigo científico completo. Mas a definição pode ser ampliada para abarcar também comentários, relatórios de resultados negativos, um conjunto de dados e sua descrição etc.

16 SCIELO Brasil, Critérios, política e procedimentos para a admissão e a permanência de periódicos na Coleção SciELO Brasil, versão maio 2020, p. 29. Disponível em: <https://wp.scielo.org/wp-content/uploads/20200500-Criterios -SciELO-Brasil.pdf>. Acesso em: 2 set. 2020.

17 Nesse sentido, em relação ao uso de preprints na história: FEITLER, Bruno. Editorial: a caminho de uma cultura de preprints? Revista Brasileira de História. São Paulo, v. 39, n. 81, p. 7-11, mai./ago. 2019, p. 9.

18 NEYLON, Cameron; PATTINSON, Damian; BILDER, Geoffrey; LIN, Jennifer. On the origin of nonequivalent states: How we can talk about preprints. F1000Research, p. 1-13, maio 2017, p. 3.

19 CHIARELLI Andrea; JOHNSON, Rob; PINFIELD, Stephen; RICHENS, Emma. Preprints and Scholarly Communication: An Exploratory Qualitative Study of Adoption, Practices, Drivers and Barriers [version 2]. F1000Research, p. 1-74, nov. 2019, p. 4-5. 
b) Tempo da publicização. São disponibilizados antes da publicação formal em um periódico científico.

c) Versão do texto. Relaciona-se com a revisão por pares, pois são versões disponibilizadas antes ou paralelamente à submissão para um periódico científico. Porém, há quem entenda que também podem abranger artigos rejeitados na revisão por pares, mas que os autores tenham interesse, ainda assim, em disponibilizar.

d) Acessibilidade. Os manuscritos são disponibilizados em servidores abertos ao público e de acesso gratuito.

e) Responsabilidade pela publicização. São publicizados ou compartilhados pelos próprios autores do trabalho.

f) Valor do trabalho para a comunidade. São manuscritos que não passaram pelo processo de publicação completo, mas que são valiosos e merecem ser facilmente acessados pela comunidade.

Há também alguma controvérsia sobre a que se refere o prefixo "pre" de preprint. Alguns autores o relacionam com a publicação formal em um periódico, de modo que se trataria de um artigo disponibilizado antes da publicação oficial ("impressão" da revista), uma "pré-publicação". ${ }^{20}$ Essa definição, contudo, abarcaria mesmo o postprint, já que este é também disponibilizado antes da publicação oficial em um periódico. Por isso, pode-se encontrar a utilização do termo preprint de um modo genérico, incluindo também o que definimos em seguida como postprint.

É mais comum, no entanto, que o prefixo "pre" seja vinculado com o processo de revisão por pares, em oposição ao "post" de postprint. Assim, o primeiro diria respeito a um artigo publicizado à comunidade antes do controle pelos pares, e o segundo após esse controle. ${ }^{21}$ Seguindo

20 LARIVIÈRE, Vincent et al. arXiv E-prints and the journal of record: An analysis of roles and relationships. Journal of the Association for Information Science and Technology, v. 65, n. 6, 1157-1169, jan. 2014, p. 1158.

21 CHIARELLI Andrea; JOHNSON, Rob; PINFIELD, Stephen; RICHENS, Emma. Preprints and Scholarly Communication: An Exploratory Qualitative Study of Adoption, Practices, Drivers and Barriers [version 2]. F1000Research, p. 1-74, nov. 2019, p. 5. 
o entendimento majoritário, bem como de documentos oficias de Scielo ${ }^{22}$ e $\mathrm{COPE}^{23}$, relacionamos os prefixos "pre" e "post" com a revisão por pares e propomos as seguintes definições:

- Preprint é a publicização pelo autor, em servidores reconhecidos, de uma versão de trabalho científico antes ou paralelamente à submissão ao controle formal por pares em uma revista científica. Ou seja, um rascunho do manuscrito antes de ser avaliado por outros pesquisadores.

- Postprint é a publicização pelo autor, em servidores reconhecidos, de uma versão de trabalho científico após ser submetido a controle formal por pares em revista científica, já com a validação e os aprimoramentos oriundos das rodadas de correções, mas antes de sua publicação oficial na revista. Normalmente costuma-se denominar tal momento como "no prelo".

Os pontos mais polêmicos dizem respeito ao preprint, pois, como veremos, a maior gama de problemas dessa prática liga-se à ausência de controle formal pelos pares. No postprint, o artigo já passou pela revisão por outros pesquisadores, o que evita a maior parte das objeções. A principal vantagem do postprint está em não se ter de aguardar a publicação oficial pelo periódico, o que pode demorar em razão, por exemplo, do número elevado de artigos na fila, de parâmetros de exogenia e titulação da autora e do autor, bem como prioridades temáticas da revista. Contudo, os resultados não são publicizados tão imediatamente como no preprint, pois se deverá aguardar ao menos as rodadas de correções pelos avaliadores.

22 “Preprints são manuscritos prontos para submissão a um periódico que são depositados em um servidor Web de acesso aberto previamente ou em paralelo à submissão a um periódico para avaliação com vistas à sua publicação formal como artigo revisado por pares." (SCIELO Brasil, Critérios, política e procedimentos para a admissão e a permanência de periódicos na Coleção SciELO Brasil, versão maio 2020, p. 29. Disponível em: <https://wp.scielo.org/ wp-content/uploads/20200500-Criterios-SciELO-Brasil.pdf>. Acesso em: 2 set. 2020).

23 "A preprint is a scholarly manuscript posted by the author(s) in an openly accessible platform, usually before or in parallel with the peer review process." (COPE Council. COPE Discussion document: Preprints. Mar. 2018, p. 2. Disponível em: <https://publicationethics.org/resources/discussion-documents/ preprints $>$. Acesso em: 16 set. 2020). 
Compreendido um mínimo conceitual em relação a tais categorias, por que devemos adotar (ou ao menos autorizar) o depósito de preprints e postprints no Direito e nas ciências criminais? Especificamente, os preprints e postprints têm benefícios como (a) a disseminação antecipada e rápida de resultados, (b) a garantia da originalidade do conteúdo, (c) a possibilidade de revisão e aprimoramento do trabalho, (d) a maior acessibilidade e (e) a publicização de conteúdos que, de outra forma, não seriam compartilhados.

Em uma sociedade em que informações circulam de forma cada vez mais acelerada, (a) a divulgação antecipada e rápida dos resultados de pesquisas científicas torna-se muito importante. ${ }^{24}$ Isso é particularmente útil nas ciências médicas, no combate a epidemias. ${ }^{25}$ Desde o início da pandemia do Covid-19, por exemplo, houve um elevado número de publicações sobre o tema, que aumentou drasticamente a velocidade dos processos editoriais em periódicos de medicina e também o número de preprints publicados na área. ${ }^{26}$ Ademais, afirma-se que uma maior celeridade na divulgação de pesquisas contribui em muito para o surgimento e a consolidação de jovens pesquisadores, visto que o sistema tradicional finda por dificultar o avanço das carreiras e manter as relações hierárquicas estratificadas no meio científico. ${ }^{27}$

24 Existem, por outro lado, críticas a uma ciência demasiadamente acelerada, com pouco tempo para reflexão. Cf., por exemplo, o Slow Science Manifesto (http://slow-science.org/).

25 JOHANSSON, Michael A.; REICH, Nicholas G.; MEYERS, Lauren A; LIPSIT$\mathrm{CH}$, Marc. Preprints: An underutilized mechanism to accelerate outbreak science. PLoS Medicine, v. 15, n. 4, abr. 2018; CHUNG, Kyu Jin. Preprints: What is their role in medical journals? Archives of Plastic Surgery, v. 47, n. 2, p. 115-117, mar. 2020, p. 115.

26 Sobre isso: HORBACH, Serge P.J.M. Medical journals drastically speed up their publication process for Covid-19. BioRxiv (preprint), abr. 2020; MAJUMDER, Maimuna; MANDL, Kenneth. Early in the epidemic: impact of preprints on global discourse about COVID-19 transmissibility. The Lancet, v. 8, e627-e630, 2020.

27 SARABIPOUR, Sarvenaz; DEBAT, Humberto J.; EMMOTT, Edward; BURGESS, Steven J.; SCHWESSINGER, Benjamin; HENSEL, Zach. On the value of preprints: An early career researcher perspective. PLoS Biology, v. 17, n. 2, e3000151, p. 1-12, 2019. 
O processo editorial tradicional toma, inevitavelmente, um certo tempo. Ainda que se adotem todas as medidas para tentar assegurar celeridade, um prazo de 90-120 dias da submissão à publicação é uma expetativa razoável (e praticamente inexistente no cenário atual das revistas científicas de Direito no Brasil, em que é comum aguardar 6, 12 meses ou mais pela publicação, com poucas exceções). ${ }^{28}$ Um estudo na área da biologia verificou que o intervalo médio entre a publicização de artigos no servidor de preprints bioRxiv e a posterior publicação em um periódico científico era de 160 dias. ${ }^{29}$

Nas ciências jurídicas, a velocidade da publicação pode ser muito importante quando os estudos abordam temas que estão na iminência de votação pelo Poder Legislativo ou de julgamento pelo Judiciário e a espera pela publicação após a revisão por pares pode tornar o conteúdo obsoleto. Diante disso, ampliou-se a publicação de textos em sites jurídicos especializados, os quais, embora certamente se caracterizem como ótimos meios de divulgação, não são certificados como fontes científicas em termos estritos, devendo-se evitar a citação em trabalhos acadêmicos, por exemplo. Assim, a ampliação dos preprints em servidores certificados para tanto pode ser um caminho adequado para reduzir o problema da demora na publicação, mantendo-se o rigor e a integridade científicos.

O compartilhamento antecipado também é relevante para (b) garantir o reconhecimento da originalidade do conteúdo, especialmente em temas que ganham especial destaque e recebem muita atenção da

28 É certo que os periódicos que conseguirem reduzir os prazos do processo editorial e acelerar a publicação dos artigos poderão evitar muitos dos problemas que dão causa à adoção de preprints. Contudo, vale ressaltar que mesmo prazos bastante curtos (p. ex., 60 dias para publicação desde o recebimento do artigo pelo periódico) poderão ser prejudiciais quando houver urgência na divulgação de resultados sobre um determinado tema. Portanto, mesmo em um cenário ideal de aceleração geral do processo editorial dos periódicos, os preprints ainda poderão ter um papel importante. How quickly do preprints become published articles? In: POWELL, Andrea; JOHNSON, Rob; HERBERT, Rachel. ICSR Perspectives. Elsevier, 2019, p. 2. Outra pesquisa aponta mediana semelhante de 174 dias entre o depósito do preprint e a publicação em revista (FU, Darwin Y; HUGHEY, Jacob J. Releasing a preprint is associated with more attention and citations for the peer-reviewed article. eLife, v. 8, e52646, p. 1-12, 2019, p. 2). 
comunidade científica. Por vezes, esperar pelo término do processo editorial tradicional pode fazer com que a ideia deixe de ser original, em razão da publicação prévia de outros artigos sobre o mesmo tópico. ${ }^{30}$ Assim, evita-se a duplicidade de pesquisas que possam ser desnecessárias, ao passo que os esforços científicos poderiam ser direcionados a avançar sobre o resultado já obtido. ${ }^{31}$

Apontam-se também benefícios na (c) revisão do conteúdo pelo autor, já que é possível alterar o artigo depois de publicizado. Os autores podem receber contribuições e críticas da comunidade científica a serem integradas a versões posteriores, o que é facilitado em alguns servidores, que possibilitam comentários aos artigos. ${ }^{32}$ Haveria, assim, uma "revisão por pares informal", ${ }^{33}$ aberta e transparente. Desse modo, a versão publicada ao final em uma revista terá sido submetida a uma análise mais ampla do meio acadêmico.

Haveria ainda vantagens de (d) acessibilidade e de impacto, uma vez que os resultados de pesquisas são acessíveis de forma gratuita a todos que tenham interesse, diferentemente de muitos periódicos, cuja assinatura ou aquisição dos artigos é custosa. ${ }^{34}$ Portanto, a publicização prévia dos trabalhos científicos está de acordo com as diretrizes de uma ciência aberta. ${ }^{35}$

30 CHIARELLI Andrea; JOHNSON, Rob; PINFIELD, Stephen; RICHENS, Emma. Preprints and Scholarly Communication: An Exploratory Qualitative Study of Adoption, Practices, Drivers and Barriers [version 2]. F1000Research, p. 1-74, nov. 2019, p. 5.

31 SOUZA, Jonathan R. S. The emergence of preprints for Brazilian science: considerations from the Nursing area. Revista da Escola de Enfermagem da USP, v. 53, e03534, p. 1-8, 2019. p. 3.

32 HOY, Matthew B. Rise of the Rxivs: How Preprint Servers are Changing the Publishing Process. Medical Reference Services Quarterly, v. 39, n. 1, p. 84-89, fev. 2020, p. 85.

33 PINFELD, Stephen. Self-archiving publications. In: GORMAN, G.E.; ROWLAND, Fytton (eds). International Yearbook of Library and Information Management 2004-2005: Scholarly publishing in an electronic era. London: Facet, 2004, p. 2.

34 HOY, Matthew B. Rise of the Rxivs: How Preprint Servers are Changing the Publishing Process. Medical Reference Services Quarterly, v. 39, n. 1, p. 84-89, fev. 2020, p. 86.

35 Sobre isso, ver: CAPPARELLI, Bruna; GIACOMOLLI, Nereu J. Editorial: Instrumentos e práticas para a Ciência aberta no Direito processual 
Afirma-se que isso possibilitaria uma maior difusão do conteúdo, com potencial aumento do impacto. Nesse sentido, pesquisas empíricas constataram que trabalhos disponibilizados em servidores de preprint e depois publicados em revistas científicas possuem maior impacto, ou seja, são mais lidos e citados do que aqueles não depositados previamente. ${ }^{36}$

Por fim, (e) viabiliza-se a publicação de textos que, de outra forma, não seriam aceitos em periódicos que passam por revisão por pares, mas que podem ser importantes para o avanço colaborativo da ciência, como pesquisas que não conseguem comprovar empiricamente suas hipóteses, réplicas de outras experimentos e resultados contraditórios. ${ }^{37}$

\section{Riscos E QUESTÕES QUE DEVEM SER PONDERADAS NA IMPLEMENTAÇÃO DE PREPRINTS}

O problema mais comumente apontado em relação aos preprints é a ausência de comprovação de qualidade do texto, em razão de não passar

penal - perplexidades e algumas possibilidades. Revista Brasileira de Direito Processual Penal, Porto Alegre, vol. 4, n. 1, p. 25-40, jan./abr. 2018.

Uma pesquisa que examinou 2.765 artigos publicados em periódicos da matemática entre 1997 e 2005 constatou que aqueles publicizados no arXiv receberam em média 35\% mais citações do que aqueles não depositados no servidor (Cf. DAVIS, Philip M.; FROMERTH, Michael J. Does the arXiv lead to higher citations and reduced publisher downloads for mathematics articles? Scientometrics, v. 71, n. 2, p. 203-215, maio 2007). Outra pesquisa mais recente analisou 74.239 artigos publicados em 39 revistas, dos quais 5.405 tinham anteriormente sido depositados como preprint, e constatou que artigos disponibilizados como preprint possuíam, em média, $49 \%$ maior Altmetric Attention Score e 36\% mais citações (Cf. FU, Darwin Y; HUGHEY, Jacob J. Releasing a preprint is associated with more attention and citations for the peer-reviewed article. eLife, v. 8, e52646, p. 1-12, 2019). Também nesse sentido: FRASE, Nicholas; MOMENI, Fakhri; MAYR, Philipp; PETERS, Isabella. The relationship between bioRxiv preprints, citations and altmetrics. Quantitative Science Studies, v. 1, n. 2, p. 618-638, 2020.

TEIXEIRA DA SILVA, Jaime A. The preprint debate: What are the issues? Medical Journal Armed Forces India, v. 74, n. 2, p. 162-164, abr. 2018, p. 164; HOY, Matthew B. Rise of the Rxivs: How Preprint Servers are Changing the Publishing Process. Medical Reference Services Quarterly, v. 39, n. 1, p. 84-89, fev. 2020, p. 86. 
por uma revisão por pares nos moldes tradicionais. ${ }^{38}$ Assim, haveria o risco de um rebaixamento dos standards de qualidade da produção científica, ${ }^{39}$ bem como a criação de incentivos aos acadêmicos para fazerem pesquisas e produzirem artigos de baixa qualidade acerca de tópicos da moda. ${ }^{40}$

Vale destacar, contudo, que em algumas plataformas, como a "arXiv" há um breve e superficial controle preliminar (desk review) dos artigos, a fim de verificar se o conteúdo não é inapropriado e se é plausível para ser de interesse para comunidade ${ }^{41}$ Em servidores relacionados à biologia e às ciências médicas (como o bioRxiv e o medRxiv), há normalmente uma revisão um pouco mais detida, em duas fases. Primeiro os artigos são examinados por um membro interno, a fim de verificar se há plágio ou incompletude. Em um segundo momento, são analisados por voluntários, especialistas no tópico do artigo, que avaliam a possível existência de conteúdo não científico ou de riscos à saúde ou à biossegurança. Podem, ainda, marcar os manuscritos para checagem futura. De todo modo, o objetivo desse controle preliminar é apenas identificar defeitos evidentes, não de avaliar a qualidade do artigo, como na revisão por pares tradicional. ${ }^{42}$

38 CHUNG, Kyu Jin. Preprints: What is their role in medical journals? Archives of Plastic Surgery, v. 47, n. 2, p. 115-117, mar. 2020, p. 116.

39 CHIARELLI Andrea; JOHNSON, Rob; PINFIELD, Stephen; RICHENS, Emma. Preprints and Scholarly Communication: An Exploratory Qualitative Study of Adoption, Practices, Drivers and Barriers [version 2]. F1000Research, p. 1-74, nov. 2019, p. 6.

40 TEIXEIRA DA SILVA, Jaime A. The preprint wars. AME Medical Journal, v. 2, n. 6, jun. 2017, p. 1-2.

41 HOY, Matthew B. Rise of the Rxivs: How Preprint Servers are Changing the Publishing Process. Medical Reference Services Quarterly, v. 39, n. 1, p. 84-89, fev. 2020, p. 86; STERLING, Evan. The Rising Popularity of Preprints. In: WATSON, Jada (ed.). Moving through the Grey: Publishing in Action. Ottawa, 2018, p. 49. Disponível em: <https://ojs.scholarsportal.info/ottawa-school/ index.php/PA/issue/view/18>. Acesso em: 02 set. 2020; COPE Council. COPE Discussion document: Preprints. Mar. 2018, p. 3. Disponível em: $<$ https://publicationethics.org/resources/discussion-documents/preprints $>$. Acesso em: 16 set. 2020.

42 KWON, Diana. How swamped preprint servers are blocking bad coronavirus research. Nature, v. 581, n. 7807, maio 2020, p. 130-131. 
Uma outra objeção aos preprints é a possível sobrecarga de informações, com publicação de um grande número de artigos desinteressantes e inúteis. ${ }^{43}$ Por exemplo, em relação ao Covid-19, os servidores bioRxiv e medRxiv publicaram mais de três mil estudos sobre o tema desde o início da pandemia. ${ }^{44}$

Informações equivocadas e não revisadas por pares podem ser divulgadas na mídia e alcançar um público que não teria condições de julgar a qualidade da produção científica ${ }^{45}$ - o que poderia fornecer uma ilegítima aparência de cientificidade a "fake news" e gerar conteúdo que alimenta teorias conspiratórias. ${ }^{46}$ Portanto, a mídia deve também diferenciar os preprints de artigos publicados após revisão por pares. ${ }^{47}$

A publicização prévia de versões dos trabalhos também pode gerar problemas para os autores no que diz respeito à exigência de ineditismo, tradicionalmente requerida pelos periódicos científicos. Assim, as revistas poderiam não aceitar artigos previamente compartilhados em servidores de preprint..$^{48}$ Essa questão assume especial importância nas ciências criminais - e no direito de modo geral -, uma vez que a maioria dos periódicos não admitem trabalhos que tenham sido anteriormente publicados como preprint ou postprint (por exemplo, RBCCrim e REC). ${ }^{49}$

43 VALE, Ronald D. Accelerating Scientific Publication in Biology. Proceedings of the National Academy of Sciences, v. 112, n. 44, p. 13439-13446, nov. 2015, p. 13444.

44 KWON, Diana. How swamped preprint servers are blocking bad coronavirus research. Nature, v. 581, n. 7807, maio 2020, p. 130-131.

45 SHELDON, Tom. The Impact of Preprint on Media Reporting of Science. The Lancet, v. 392, n. 10154, out. 2018, p. 1194.

46 KWON, Diana. How swamped preprint servers are blocking bad coronavirus research. Nature, v. 581, n. 7807, maio 2020, p. 130-131.

47 HOY, Matthew B. Rise of the Rxivs: How Preprint Servers are Changing the Publishing Process. Medical Reference Services Quarterly, v. 39, n. 1, p. 84-89, fev. 2020, p. 86.

48 NALLAMOTHU, Brahmajee K.; HILL; Joseph A. Preprints and Cardiovascular Science Prescient or Premature? Circulation, v. 136, n. 13, p. 1177-1179, set. 2017, p. 1178; HOY, Matthew B. Rise of the Rxivs: How Preprint Servers are Changing the Publishing Process. Medical Reference Services Quarterly, v. 39, n. 1, p. 84-89, fev. 2020, p. 87.

49 “1.1.b) O artigo deve ser inédito; portanto, não publicado em nenhum outro periódico científico e/ou canais de comunicação. Vale ressaltar que 
Trata-se, em grande medida, de uma questão política em prol da ciência aberta ${ }^{50}$ e que também envolve complexidades econômicas aos periódicos, cujo futuro dependerá de uma tendência geral de aceitação de preprints por parte das revistas. Por enquanto, é de extrema importância que os autores consultem antes as normas de cada periódico antes de depositar a pesquisa em servidor, o que pode ser feito em diretórios de políticas editoriais de revistas científicas, como Sherpa Romeo e Diadorim.

Por fim, vale notar que os preprint normalmente não entram em métricas científicas oficiais. ${ }^{51}$ Por exemplo, os servidores de preprint não têm fator de impacto ${ }^{52}$ e os artigos neles publicizados geralmente não são considerados em pontuações para concursos para docência, seleções de bolsas e outros editais, embora tal cenário possa se alterar futuramente..$^{53}$ Além disso, discute-se a possibilidade de citação a um preprint em pes-

ineditismo não deve ser confundido com originalidade, podendo o tema tratado pelo artigo já ter sido abordado por outros autores(as) e/ou publicações” (Políticas Editoriais RBCCRIM. Disponível em: <https://www.ibccrim.org. br/publicacoes/pagina/7>. Acesso em: 19 set. 2020). “1.2 Os textos enviados deverão ser inéditos no Brasil, independente da forma de publicação, impressa e/ou eletrônica, e sendo vedada a análise de artigos submetidos simultaneamente a mais de um periódico" (Políticas Editoriais REC. Disponível em: <http://www.itecrs.org/edicoes/regras:ler>. Acesso em: 19 set. 2020).

50 CHIARELLI Andrea; JOHNSON, Rob; PINFIELD, Stephen; RICHENS, Emma. Preprints and Scholarly Communication: An Exploratory Qualitative Study of Adoption, Practices, Drivers and Barriers [version 2]. F1000Research, p. 1-74, nov. 2019, p. 6; FERREIRA, Carlos M.; SERPA, Sandro. The Importance of Preprint in Scientific Publication: Perspectives and Challenges. The Journal of Social Sciences Research, v. 4, n. 12, p. 642-647, 2018. p. 643.

51 Vale notar que, embora não ingressem em indexadores controlados (como Scopus e WoS), os preprints podem ter o número de citações calculado pelo índice $\mathrm{H}$ do Google Acadêmico, o que para algumas áreas do conhecimento, como o Direito, seria valorado. Ainda não se sabe exatamente como isso se desenvolverá, contudo se aponta a tendência de que as citações aos preprints não seriam computadas às revistas em que possam ser posteriormente publicados, mas ao próprio servidor de preprint.

52 ELMORE, Susan A. Preprints: What Role Do These Have in Communicating Scientific Results? Toxicologic Pathology, v. 46, n. 4, p. 364-365, abr. 2018, p. 364.

53 MAGGIO, Lauren; ARTINO JR., Anthony; DRIESSEN, Erik. Preprints: Facilitating early discovery, access, and feedback. Perspectives on Medical Education, v. 7, p. 287-289, 2018. p. 287. 
quisas científicas, visto que não publicados em revistas certificadas e com controle por pares. ${ }^{54}$ Esta é uma questão pertinente, a ser ponderada com cautela em discussões sobre implementação de preprints. Preliminarmente, pensa-se que a citação pode ser aceita se ainda não publicada a versão final em revista, porém sempre com identificação na referência de que se trata de preprint e atenção redobrada em relação à confiabilidade da pesquisa. ${ }^{55}$

\section{IMPACTOS SOBRE O PROCESSO EDITORIAL E SOBRE A PRODUÇÃO CIENTÍFICA EM GERAL}

Os preprints afetam o processo editorial tradicional em dois pontos que historicamente são tidos como fundamentos importantes na produção científica: (a) o ineditismo e (b) a avaliação dupla-cega por pares. ${ }^{56} \mathrm{Tal}$ discussão ressalta um conflito entre dois princípios caros para o movimento da ciência aberta: a disseminação do conhecimento e a validação por pares. Assim, faz-se necessário que a comunidade científica discuta normas e procedimentos a fim de esclarecer o estatuto dos documentos, não apenas para os seus membros, mas também para o público externo. É inegável, ainda, que se torna mais complexo identificar o grau de cientificidade dos textos aos quais se tem acesso. ${ }^{57}$

54 CHIARELLI Andrea; JOHNSON, Rob; PINFIELD, Stephen; RICHENS, Emma. Preprints and Scholarly Communication: An Exploratory Qualitative Study of Adoption, Practices, Drivers and Barriers [version 2]. F1000Research, p. 1-74, nov. 2019. p. 12.

55 Ressaltando a ampliação da responsabilidade do pesquisador para verificar a qualidade do que está citando: FERREIRA, Carlos M.; SERPA, Sandro. The Importance of Preprint in Scientific Publication: Perspectives and Challenges. The Journal of Social Sciences Research, v. 4, n. 12, p. 642-647, 2018. p. 645.

56 FEITLER, Bruno. Editorial: a caminho de uma cultura de preprints? Revista Brasileira de História. São Paulo, v. 39, n. 81, p. 7-11, mai./ago. 2019, p. 9. Sobre o controle por pares, ver: VASCONCELLOS, Vinicius G. Editorial: Controle por pares e a função do revisor - premissas e orientações para uma avaliação consistente. Revista Brasileira de Direito Processual Penal, Porto Alegre, vol. 3, n. 2, p. 437-458, mai./ago. 2017.

57 STERLING, Evan. The Rising Popularity of Preprints. In: WATSON, Jada (ed.). Moving through the Grey: Publishing in Action. Ottawa, 2018, p. 57. 
Primeiramente, (a) consolida-se uma "nova" função dos periódicos científicos em razão da mudança sobre a perspectiva do requisito de ineditismo. Os artigos disponibilizados em servidores certificados de preprint passam a ser aceitos como contribuições originais (não duplicadas ou redundantes, ou seja, que aportam um avanço no estado atual da discussão científica sobre o tema concreto), embora não inéditos em termos estritos. ${ }^{58}$ Assim, em tais casos há uma alteração na lógica editorial, de modo que a publicação em revista científica passa a ser um certificado de "confiabilidade e qualidade" por meio da validação pelos pares, ${ }^{59}$ e não um meio para a sua divulgação inicial e primordial.

Desse modo, os artigos publicizados como preprints e publicados em periódicos científicos têm propósitos distintos. O compartilhamento em servidores antes da revisão tem como finalidade disponibilizar as informações de forma célere à comunidade científica, em razão dos benefícios anteriormente indicados. Por outro lado, a publicação em periódicos reconhecidos e com controle pelos pares serve para assegurar a qualidade do artigo e avaliar a produção de um pesquisador, bem como a confiabilidade e reprodutibilidade dos dados. ${ }^{60}$

Disponível em: <https://ojs.scholarsportal.info/ottawa-school/index.php/ PA/issue/view/18>. Acesso em: 02 set. 2020.

"Preprints are not considered prior publication in a way that would prevent later publication after peer review in a journal" COPE Council. COPE Discussion document: Preprints. Mar. 2018, p. 3. Disponível em: <https://publicationethics.org/resources/discussion-documents/preprints>. Acesso em: 16 set. 2020.

59 "A função chave do periódico passa a ser a de validar a pesquisa como revisada por pares." SCIELO Brasil, Critérios, política e procedimentos para a admissão e a permanência de periódicos na Coleção SciELO Brasil, versão maio 2020, p. 29. Disponível em: <https://wp.scielo.org/wp-content/uploads/20200500-Criterios-SciELO-Brasil.pdf>. Acesso em: 02 set. 2020. Sobre isso, ver: VASCONCELLOS, Vinicius G. Editorial: a função do periódico científico e do editor para a produção do conhecimento no Direito e nas ciências criminais. Revista Brasileira de Direito Processual Penal, Porto Alegre, vol. 3, n. 1, p. 9-17, jan./ abr. 2017; MINORELLI, Lucas; SILVA, Willians M. Publicações em periódicos de ciências criminais no Brasil: uma perspectiva de editores. Revista dos Tribunais, São Paulo, v. 1009, p. 359-381, nov. 2019.

60 ELMORE, Susan A. Preprints: What Role Do These Have in Communicating Scientific Results? Toxicologic Pathology, v. 46, n. 4, p. 364-365, abr. 2018, p. 
Percebe-se, portanto, que a implementação de sistemas de preprint não acarretará o fim das revistas científicas. Em realidade, poderá aprimorá-las, pois haverá a consolidação do papel de verificação e certificação realizado pelos periódicos, com a otimização de seus fluxos e do próprio processo de avaliação em razão da adoção de práticas de ciência aberta. ${ }^{61}$

Além disso, como ponto fundamental, a publicação em uma revista certificada possibilita a inclusão do artigo em repositórios e indexadores de referência, os quais são fontes necessárias para levantamento bibliográfico em outras pesquisas. Em um cenário de produção intensa de textos e fácil acesso pela internet, a existência de indexadores e catálogos (como Scopus, WoS, Scielo, etc.) serve exatamente para oferecer uma fonte segura de textos de qualidade, submetidos a um controle para seleção criteriosa. Ou seja, em um mundo de textos facilmente acessíveis pela internet, uma pesquisa deve necessariamente ao menos citar os artigos sobre o tema que estejam disponíveis em indexadores certificados.

Como segunda consequência ao processo editorial tradicional, (b) ocasiona-se a identificação da autoria do trabalho antes de sua avaliação formal. Ao postar o artigo no servidor de preprint, ele se torna público antes do controle por pares e, assim, resta inviável assegurar o anonimato do/a autor/a aos/às pareceristas. ${ }^{62}$ Ou seja, nesses casos altera-se o sistema de controle duplo-cego para simples-cego por pares, onde avaliadores poderão descobrir a identidade do/a autor/a, mas autor/a não saberá quem são os avaliadores. ${ }^{63}$

364; BERG, Jeremy M. et al. Preprints for the life sciences. Science, v. 352, n. 6288, p. 899-901, maio 2016.

61 FERREIRA, Carlos M.; SERPA, Sandro. The Importance of Preprint in Scientific Publication: Perspectives and Challenges. The Journal of Social Sciences Research, v. 4, n. 12, p. 642-647, 2018. p. 644.

SCIELO Brasil, Critérios, política e procedimentos para a admissão e a permanência de periódicos na Coleção SciELO Brasil, versão maio 2020, p. 29. Disponível em: <https://wp.scielo.org/wp-content/uploads/20200500-Criterios -SciELO-Brasil.pdf>. Acesso em: 02 set. 2020.

${ }_{63}$ Sobre isso: SOUZA, Jonathan R. S. The emergence of preprints for Brazilian science: considerations from the Nursing area. Revista da Escola de Enfermagem da USP, v. 53, e03534, p. 1-8, 2019. p. 4. 
Pensa-se que a revisão duplo-cega ainda pode ser considerada um procedimento importante para buscar a imparcialidade das avaliações. Por essa razão, o depósito do preprint deve ser uma opção autorizada, mas não exigida. ${ }^{64} \mathrm{Ou}$ seja, produto de um ato unilateral do autor, que poderá decidir se quer publicizar seu artigo antes do controle pelos pares, identificando a autoria, ou mantê-lo secreto, garantindo o anonimato no momento da avaliação. Com isso, garante-se a possibilidade de que o autor, se quiser, não seja identificado, mas também se oferecem as potencialidades do preprint.

\section{CONSIDERAÇÕES FINAIS}

Diante do exposto, conclui-se que a tendência de ampliação de preprints aporta um cenário de novas perspectivas à produção de conhecimento e à editoração de revistas científicas, contribuindo com a consolidação de medidas para uma ciência aberta. Seus riscos e benefícios devem ser ponderados com cautela, especialmente diante dos impactos em premissas tradicionais do processo editorial científico. Contudo, pensa-se que os potenciais benefícios se sobressaem:

a) Por um lado, permite-se a divulgação dos resultados da pesquisa rapidamente, especialmente em questões urgentes (casos em julgamento nos Tribunais, novas alterações legislativas, etc.). A demora inerente ao controle por pares costuma afastar autores que preferem publicar suas pesquisas imediatamente em sites não certificados como revistas científicas. Assim, a adoção de preprints pode alterar tal panorama e potencializar o impacto das investigações.

b) Além disso, possibilita-se um debate amplo, transparente e prévio à publicação, o que pode resultar em aprimoramentos das pesquisas até a sua publicação final. No todo, tal mudança não prejudica a

64 "O uso dos preprints é uma opção e escolha dos autores e cabe aos periódicos adaptarem suas políticas para aceitar a submissão de manuscritos previamente depositados em um servidor de preprint reconhecido pelo periódico." (SCIELO Brasil, Critérios, política e procedimentos para a admissão e a permanência de periódicos na Coleção SciELO Brasil, versão maio 2020, p. 7. Disponível em: <https://wp.scielo.org/wp-content/uploads/20200500-Criterios-SciELO-Brasil.pdf>. Acesso em: 02 set. 2020). 
qualidade e a seriedade do controle por pares e do processo editorial em geral, mas traz importantes benefícios e avanços para o campo científico.

c) A ampliação da publicidade e da divulgação das pesquisas de um modo célere potencializa a interação entre investigadores e grupos de pesquisa em temas correlatos e semelhantes, o que contribui para $o$ desenvolvimento científico da área e tende a evitar esforços desperdiçados em resultados duplicados. Ademais, contribui para o avanço da ciência aberta a partir da adoção de servidores gratuitos e certificados para tanto.

Aos autores que pretendam publicizar suas pesquisas em servidores de preprint, alguns cuidados são necessários. Em um cenário de poucas revistas atualmente adeptas à política de autorização a preprint, $\mathrm{o}$ depósito do artigo pode reduzir as potenciais vias de publicação do texto, em razão da perda de ineditismo nos termos normalmente requeridos pelos periódicos. Além disso, o depósito deve ser feito em servidores certificados e, então, deve-se atentar cuidadosamente aos termos de direitos autorais previstos em cada um.

Ao realizar a submissão para uma revista, a autora deve avisar a equipe editorial sobre o depósito do artigo em servidor de preprint, apontando o DOI para verificação. Se houver a publicação do trabalho no periódico, a autora deve atualizar o preprint depositado para indicar tal publicação final com o respectivo DOI, visto que eventual citação da pesquisa deverá dirigir-se à versão final da revista.

Com a crescente exigência de celeridade na divulgação de informações, verifica-se uma tendência marcante no sentido de que os periódicos científicos passem a aceitar preprints, juntamente a outras medidas para consolidação de ciência aberta. Ademais, não se trata somente de maior celeridade, mas da consolidação de um sistema transparente e aberto de produção do conhecimento científico, o qual pode contribuir inclusive para o aprimoramento da própria pesquisa.

\section{REFERÊNCIAS}

BERG, Jeremy M. et al. Preprints for the life sciences. Science, v. 352, n. 6288, p. 899-901, maio 2016. https://doi.org/10.1126/science.aaf9133 
CAPPARELLI, Bruna; GIACOMOLLI, Nereu J. Editorial: Instrumentos e práticas para a Ciência aberta no Direito processual penal - perplexidades e algumas possibilidades. Revista Brasileira de Direito Processual Penal, Porto Alegre, vol. 4, n. 1, p. 25-40, jan./abr. 2018. https://doi.org/10.22197/rbdpp.v4i1.137

CHIARELLI Andrea; JOHNSON, Rob; PINFIELD, Stephen; RICHENS, Emma. Preprints and Scholarly Communication: An Exploratory Qualitative Study of Adoption, Practices, Drivers and Barriers [version 2]. F1000Research, p. 1-74, nov. 2019. https://doi.org/10.12688/f1000research.19619.2

CHUNG, Kyu Jin. Preprints: What is their role in medical journals? Archives of Plastic Surgery, v. 47, n. 2, p. 115-117, mar. 2020. https://doi.org/10.5999/ aps.2020.00262

COBB Matthew. The prehistory of biology preprints: a forgotten experiment from the 1960s. PLoS Biology, v. 15, n. 11, nov. 2017, n.p. https://doi.org/10.1371/ journal.pbio.2003995

COPE Council. COPE Discussion document: Preprints. Mar. 2018. Disponível em: <https://publicationethics.org/resources/discussion-documents/preprints>. Acesso em: 16 set. 2020.

DAVIS, Philip M.; FROMERTH, Michael J. Does the arXiv lead to higher citations and reduced publisher downloads for mathematics articles? Scientometrics, v. 71, n. 2, p. 203-215, maio 2007.

ELMORE, Susan A. Preprints: What Role Do These Have in Communicating Scientific Results? Toxicologic Pathology, v. 46, n. 4, p. 364-365, abr. 2018, p. 364. https://doi.org/10.1177/0192623318767322

FEITLER, Bruno. Editorial: a caminho de uma cultura de preprints? Revista Brasileira de História. São Paulo, v. 39, n. 81, p. 7-11, mai./ago. 2019. http://dx.doi. org/10.1590/1806-93472019v39n81-00

FERREIRA, Carlos M.; SERPA, Sandro. The Importance of Preprint in Scientific Publication: Perspectives and Challenges. The Journal of Social Sciences Research, v. 4, n. 12, p. 642-647, 2018. https://doi.org/10.32861/jssr.412.642.647

FU, Darwin Y; HUGHEY, Jacob J. Releasing a preprint is associated with more attention and citations for the peer-reviewed article. eLife, v. 8, e52646, p. 1-12, 2019. https://doi.org/10.7554/eLife.52646

FRASE, Nicholas; MOMENI, Fakhri; MAYR, Philipp; PETERS, Isabella. The relationship between bioRxiv preprints, citations and altmetrics. Quantitative Science Studies, v. 1, n. 2, p. 618-638, 2020. https://doi.org/ 10.1162/qss_a_00043 
GENTIL-BECCOT, Anne; MELE; Salvatore; Brooks, Travis C. Citing and Reading Behaviours in High-Energy Physics. Scientometrics, v. 84, n. 2, p. 345-355, ago. 2010, p. 347-8. https://doi.org/10.1007/s11192-009-0111-1

HERBERT, Rachel; GASSON, Kate; PONSFORD, Alex. The Need for Speed, How quickly do preprints become published articles? In: POWELL, Andrea; JOHNSON, Rob; HERBERT, Rachel. ICSR Perspectives. Elsevier, 2019.

HORBACH, Serge P.J.M. Medical journals drastically speed up their publication process for Covid-19. BioRxiv (preprint), abr. 2020. https://doi. org/10.1101/2020.04.18.045963

HOY, Matthew B. Rise of the Rxivs: How Preprint Servers are Changing the Publishing Process. Medical Reference Services Quarterly, v. 39, n. 1, p. 84-89, fev. 2020. https://doi.org/10.1080/02763869.2020.1704597

JOHANSSON, Michael A.; REICH, Nicholas G.; MEYERS, Lauren A; LIPSITCH, Marc. Preprints: An underutilized mechanism to accelerate outbreak science. PLoS Medicine, v. 15, n. 4, abr. 2018. https://doi.org/10.1371/journal.pmed.1002549

KWON, Diana. How swamped preprint servers are blocking bad coronavirus research. Nature, v. 581, n. 7807, p. 130-131, maio 2020. https://doi.org/10.1038/ d41586-020-01394-6

LARIVIÈRE, Vincent et al. arXiv E-prints and the journal of record: An analysis of roles and relationships. Journal of the Association for Information Science and Technology, v. 65, n. 6, 1157-1169, jan. 2014. https://doi.org/10.1002/asi.23044

LUTHER, Judy. The Stars Are Aligning for Preprints. The Scholarly Kitchen, 18 de abril de 2017. Disponível em: <https://scholarlykitchen.sspnet.org/2017/04/18/ stars-aligning-preprints/>. Acesso em: 16 set. 2020.

MAJUMDER, Maimuna; MANDL, Kenneth. Early in the epidemic: impact of preprints on global discourse about COVID-19 transmissibility. The Lancet, v. 8, e627-e630, 2020. https://doi.org/10.1016/S2214-109X(20)30113-3

MAGGIO, Lauren; ARTINO JR., Anthony; DRIESSEN, Erik. Preprints: Facilitating early discovery, access, and feedback. Perspectives on Medical Education, v. 7, p. 287-289, 2018. https://doi.org/10.1007/s40037-018-0451-8

MINORELLI, Lucas; SILVA, Willians M. Publicações em periódicos de ciências criminais no Brasil: uma perspectiva de editores. Revista dos Tribunais, São Paulo, v. 1009, p. 359-381, nov. 2019. 
NALLAMOTHU, Brahmajee K.; HILL; Joseph A. Preprints and Cardiovascular Science Prescient or Premature? Circulation, v. 136, n. 13, p. 1177-1179, set. 2017. https://doi.org/10.1161/CIRCULATIONAHA.117.031238

NEYLON, Cameron; PATTINSON, Damian; BILDER, Geoffrey; LIN, Jennifer. On the origin of nonequivalent states: How we can talk about preprints. F1000Research, p. 1-13, maio 2017, p. 3. https://doi.org/10.12688/f1000research.11408.1

PINFELD, Stephen. Self-archiving publications. In: GORMAN, G.E.; ROWLAND, Fytton (eds). International Yearbook of Library and Information Management 20042005: Scholarly publishing in an electronic era. London: Facet, 2004.

SARABIPOUR, Sarvenaz; DEBAT, Humberto J.; EMMOTT, Edward; BURGESS, Steven J.; SCHWESSINGER, Benjamin; HENSEL, Zach. On the value of preprints: An early career researcher perspective. PLoS Biology, v. 17, n. 2, e3000151, p. 1-12, 2019. https://doi.org/10.1371/journal.pbio.3000151

SCIELO Brasil, Critérios, política e procedimentos para a admissão e a permanência de periódicos na Coleção SciELO Brasil, versão maio 2020, p. 7. Disponível em: <https://wp.scielo.org/wp-content/uploads/20200500-Criterios-SciELO-Brasil. pdf>. Acesso em: 02 set. 2020.

SHELDON, Tom. The Impact of Preprint on Media Reporting of Science. The Lancet, v. 392, n. 10154, p. 1194, out. 2018. https://doi.org/10.1016/ S0140-6736(18)31871-3

SOUZA, Jonathan R. S. The emergence of preprints for Brazilian science: considerations from the Nursing area. Revista da Escola de Enfermagem da USP, v. 53, e03534, p. 1-8, 2019. https://doi.org/10.1590/S1980-220X2019020803534

STERLING, Evan. The Rising Popularity of Preprints. In: WATSON, Jada (ed.). Moving through the Grey: Publishing in Action. Ottawa, 2018. Disponível em: <https://ojs.scholarsportal.info/ottawa-school/index.php/PA/issue/view/18>. Acesso em: 02 set.2020.

TEIXEIRA DA SILVA, Jaime A. The preprint debate: What are the issues? Medical Journal Armed Forces India, v. 74, n. 2, p. 162-164, abr. 2018.

TEIXEIRA DA SILVA, Jaime A. The preprint wars. AME Medical Journal, v. 2, n. 6, jun. 2017. http://dx.doi.org/10.21037/amj.2017.05.23

TENNANT, Jonathan P. et al. Ten Hot Topics around Scholarly Publishing. MDPI, v. 7, n. 2, jun. 2019. https://doi.org/10.3390/publications7020034 
TENNANT, Jonathan; BAUIN, Serge; JAMES, Sarah; KANT, Juliane. The evolving preprint landscape: Introductory report for the Knowledge Exchange working group on preprints. MetaArXiv (preprint), maio 2018. https://doi.org/10.31222/ osf.io/796tu

VALE, Ronald D. Accelerating Scientific Publication in Biology. Proceedings of the National Academy of Sciences, v. 112, n. 44, 13439-13446, nov. 2015. https://doi. org/10.1073/pnas.1511912112

VASCONCELLOS, Vinicius G. Editorial: a função do periódico científico e do editor para a produção do conhecimento no Direito e nas ciências criminais. Revista Brasileira de Direito Processual Penal, Porto Alegre, vol. 3, n. 1, p. 9-17, jan./abr. 2017. https://doi.org/10.22197/rbdpp.v3i1.34

VASCONCELLOS, Vinicius G. Editorial: Controle por pares e a função do revisor - premissas e orientações para uma avaliação consistente. Revista Brasileira de Direito Processual Penal, Porto Alegre, vol. 3, n. 2, p. 437-458, mai./ago. 2017. https://doi.org/10.22197/rbdpp.v3i2.70 


\section{Informações adicionais e declarações do autor (integridade científica)}

Agradecimentos (acknowledge): Agradecemos a Lucas Minorelli pela leitura da versão preprint deste editorial e pelas sugestões de aprimoramentos.

Declaração de conflito de interesses (conflict of interest declaration): Os autores são editores da Revista Brasileira de Direito Processual Penal. Os autores confirmam que não há conflitos de interesse na realização das pesquisas expostas e na redação deste editorial.

Declaração de autoria (declaration of authorship): Todas e somente as pessoas que atendem aos requisitos de autoria deste editorial estão listadas como autores.

- Vinicius Vasconcellos: projeto e esboço inicial (conceptualization), desenvolvimento da metodologia (methodology), levantamento bibliográfico (investigation), revisão bibliográfica (investigation), redação (writing original draft), participação ativa nas discussões dos resultados (validation), revisão crítica com contribuições substanciais (writing - review and editing), aprovação da versão final.

- Felipe De-Lorenzi: projeto e esboço inicial (conceptualization), desenvolvimento da metodologia (methodology), revisão bibliográfica (investigation), redação (writing - original draft), participação ativa nas discussões dos resultados (validation), revisão crítica com contribuições substanciais (writing - review and editing), aprovação da versão final.

Declaração de originalidade (declaration of originality): Os autores apontam que o texto aqui publicado foi depositado como preprint em 24.9.2020 (https://doi.org/10.1590/SciELOPreprints.1243). Futura republicação somente se realizará com a indicação expressa da referência desta publicação original; também atestam que não há plágio de terceiros ou autoplágio. 


\section{COMO CITAR ESTE EDITORIAL:}

VASCONCELLOS, Vinicius G.; DE-LORENZI, Felipe da Costa. Editorial Preprint e postprint em publicações científicas e no Direito: discussões e medidas para ciência aberta e divulgação de pesquisas. Revista Brasileira de Direito Processual Penal, Porto Alegre, vol. 6, n. 3, p. 1091-1116, set./dez. 2020. https://doi.org/10.22197/rbdpp.v6i3.452

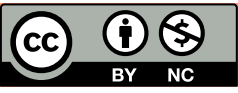

Esta obra está licenciada com uma Licença Creative Commons Atribuição-NãoComercial 4.0 Internacional. 